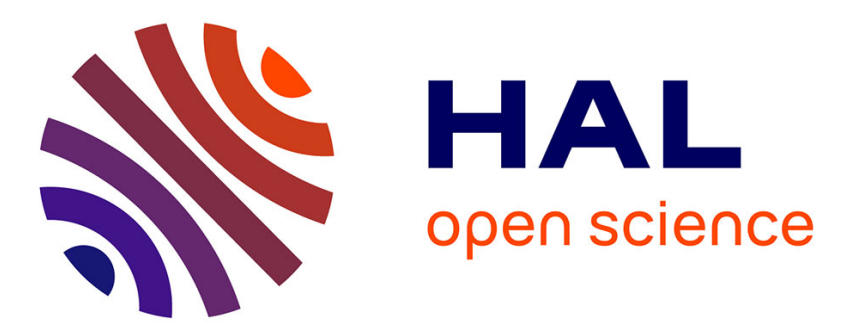

\title{
Single-shot observation of breathers from noise-induced modulation instability using heterodyne temporal imaging
}

Alexandre Lebel, Alexey Tikan, Stephane Randoux, Pierre Suret, François Copie

\section{To cite this version:}

Alexandre Lebel, Alexey Tikan, Stephane Randoux, Pierre Suret, François Copie. Single-shot observation of breathers from noise-induced modulation instability using heterodyne temporal imaging. Optics Letters, 2021, 46 (2), pp.298. 10.1364/OL.408730 . hal-03103388

\section{HAL Id: hal-03103388 \\ https://hal.science/hal-03103388}

Submitted on 8 Jan 2021

HAL is a multi-disciplinary open access archive for the deposit and dissemination of scientific research documents, whether they are published or not. The documents may come from teaching and research institutions in France or abroad, or from public or private research centers.
L'archive ouverte pluridisciplinaire HAL, est destinée au dépôt et à la diffusion de documents scientifiques de niveau recherche, publiés ou non, émanant des établissements d'enseignement et de recherche français ou étrangers, des laboratoires publics ou privés. 


\title{
Single-shot observation of breathers from noise-induced modulation instability using heterodyne temporal imaging
}

\author{
Alexandre Lebel ${ }^{1}$, Alexey Tikan $^{1}{ }^{12}$, Stephane Randou ${ }^{1}$, Pierre Suret $^{1}$, and Françols \\ COPIE $^{1, *}$ \\ ${ }^{1}$ Univ. Lille, CNRS, UMR 8523 - PhLAM - Physique des Lasers Atomes et Molécules, F-59000 Lille, France \\ ${ }^{2}$ Currently with Institute of Physics, Swiss Federal Institute of Technology Lausanne (EPFL), CH-1015 Lausanne, Switzerland \\ *Corresponding author: francois.copie@univ-lille.fr
}

Published January 8, 2021

We report phase and amplitude measurements of large coherent structures originating from the noise-induced modulation instability in optical fibers. By using a specifically-designed time-lens system (SEAHORSE) in which aberrations are compensated, the complex field is recorded in single-shot over long durations of 200 ps with sub-picosecond resolution. Signatures of Akhmediev breather-like patterns are identified in the ultrafast temporal dynamics in very good agreement with numerical predictions based on the nonlinear Schrödinger equation.

Modulation instability (MI) is unarguably one of the most ubiquitous nonlinear phenomenon since it appears in fields as different as hydrodynamics [1], plasma physics [2], BoseEinstein condensates [3] or optics [4], to name a few. MI is a long-wave instability at the origin of the amplification of arbitrary small perturbations of a homogeneous field either in space or time [5]. In the context of fiber optic experiments, a very common scenario is the so-called noise induced MI in which the instability of a continuous wave (cw) field (referred to as the pump) is triggered by the random spectral components that fall within the MI gain bandwidth [6]. Following its first observation more than 3 decades ago [4], noise induced MI in optical fibers has been the subject of numerous experimental studies and has often played a key role in the implementation of single-shot detection methods due to its intrinsic stochastic nature and fast time scale. For instance techniques like the time stretch-dispersive Fourier transformation method (TS-DFT) have revealed shot-to-shot fluctuations of the Fourier power spectrum of MI [7-10] while the so-called time lens systems have enabled the first single-shot intensity recordings of the breakup of optical fields with a picosecond timescale [11, 12]. Single-shot spatiotemporal diagrams of MI dynamics have been recently captured using a recirculating fiber loop operating at low $\mathrm{cw}$ pumping power $[13,14]$. Also, the time measurement of the amplitude and phase fluctuations of random optical wavefields has been reported in Refs. [15-17]. In particular, an heterodyne time-lens, has been used to observe the dynamics of partially coherent waves in optical fiber [17]. In this work, we use the heterodyne time-lens technique in its digital time-holography or SEAHORSE (spatial encoding arrangement with hologram observation for recording in single shot the electric field) configuration [17] to study the local emergence of breathers structures in the dynamics of the MI process in optical fiber experiments. The simultaneous measurement of the intensity and the phase of the field in single-shot over a temporal window of $200 \mathrm{ps}$ allows us to identify local emergence of quasi-periodic pulse trains accompanied with a characteristic structuration of the phase reminiscent of the Akhmediev breather.

The experimental setup exploites the SEAHORSE technique described for the first time in Ref. [17] and is schematically described in Fig. 1(a). The cw emission from single-mode fiber laser at $1550 \mathrm{~nm}$ (signal beam) is chopped down by an acousto-optic modulator (AOM) into 50 ns-long pulses to mitigate Brillouin scattering, and amplified by an Erbium-doped fiber amplifier (EDFA). It is then launched in a $500 \mathrm{~m}$-long single-mode fiber (SMF-28) where it experiences MI before entering the detection stage described hereafter. For the ultra-fast analysis of the signal beam, we used the SEAHORSE technique introduced in Ref. [17] which consists in a spectral encoding system coupled to a heterodyne measurement that allows simultaneous measurement of the intensity and phase of the field. To perform the time-lens effect, the signal beam is nonlinearly mixed with $\sim 200$ ps-long chirped pulse $(800 \mathrm{~nm}$, pump beam derived from a Coherent Astrella Ti:Sa amplifier) in a BBO crystal. From this interaction, a beam at $528 \mathrm{~nm}$ is generated via sum-frequency generation (SFG). Through this process, the ultra-fast fluctuations of the signal are encoded on the spectrum of the $528 \mathrm{~nm}$ beam. A diffraction grating ( 1600 lines $/ \mathrm{mm}$ ) is then used to map the frequency domain onto the pixels of a high-speed camera along the horizontal axis (over a 512 pixels window). Overall, this setup performs a time-to-frequency-to-space mapping that allows acquisitions over a large temporal window of $\sim 200$ ps with a resolution shorter than 1 ps. For comparison, in Ref. [17], this setup was used in a different configuration yielding a more restricted field of view of $\sim 40$ ps and a resolution of $\sim 80 \mathrm{fs}$.

In addition to the spectral encoding system, a reference beam provided by a cw laser at $1550 \mathrm{~nm}$ is used to perform a heterodyne measurement of the phase of the signal. To do so, the beams are elliptically shaped before they overlap in the BBO crystal (see Fig. 1(b) for an example of camera recording). The reference beam makes a small angle relative to the signal and pump beams resulting in horizontal fringes of interference that are recorded along the vertical axis of the camera. The relative phase between the signal beam and the reference beam is then easily retrieved by tracking the vertical position of the 


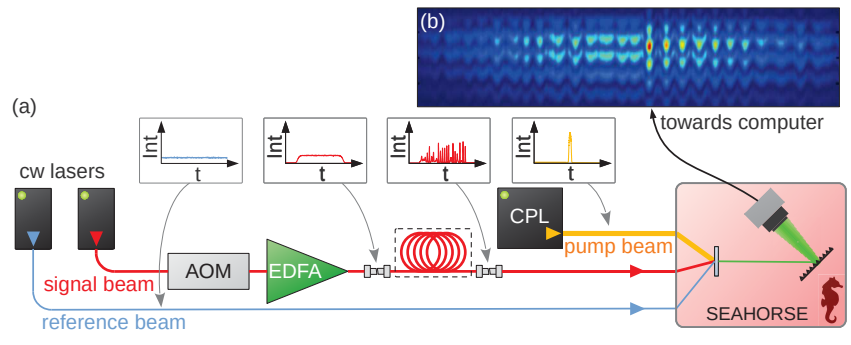

(c)

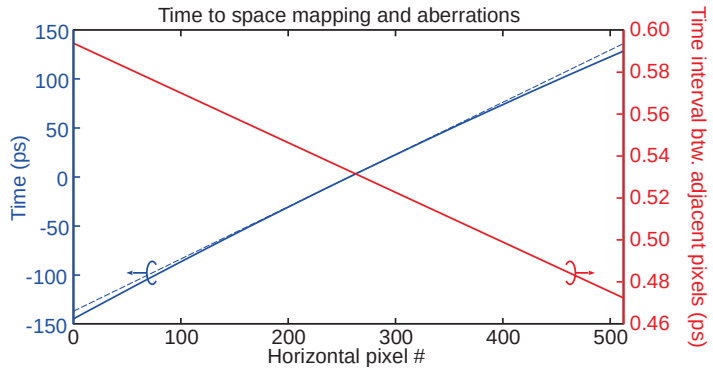

Fig. 1. (a) Schematic description of the experimental setup. (b) Example of a single-shot recording in false colors. (c) Mapping of the time on the horizontal axis of the camera taking account (solid blue line) or not (dashed blue line) of the cubic contribution to the phase of the pump pulses (red line is the derivative of the solide blue line). cw: continuous wave, AOM: acoustooptic modulator, EDFA: Erbium-doped fiber amplifier, CPL: chirped pulse laser

fringes along the horizontal axis. The angle between the beams is ajusted so that around 5 horizontal fringes are observed (Fig. $1(b))$. Note that the coherence timescale of the reference laser $(\sim 3 \mu \mathrm{s})$ is much longer than the temporal acquisition window such that the phase of the reference beam is effectively constant over each acquisition window. Frames are recorded at a $1 \mathrm{kHz}$ repetition rate which is fixed by the pump laser. Numerical processing of the data is performed afterwards to retrieve both the intensity and the phase of the field of each frame.

As a consequence of the large temporal field of view in the present configuration (5 times longer than in precedently reported experiments [11, 12]), significant aberrations on the borders of the window are observed. Indeed, in analogy with the regular thin lens that affects a field by imposing a transverse quadratic phase at leading order, the time-lens effect is obtained by imposing a longitudinal quadratic phase to the signal. This is done experimentally at the stage of SFG where the chirp of the pump pulse is transfered to the $528 \mathrm{~nm}$ beam. However, it is well known that the chirp applied to pulses by the stretcher in a regenerative amplifier is not perfectly linear because of higher order dispersion effects $[18,19]$. As a consequence of this imperfect linear chirp, there is a significant cubic contribution to the phase resulting in the temporal aberrations.

In this work, we compensate the aberrations during the numerical processing of our experimental recordings for the first time to our knowledge. The principle is summarized in Fig. 1(c). In the aberration-free case, there is a linear mapping between time and camera pixel coordinate (dashed blue line). However, the cubic contribution to the phase of the pump leads to a nonlinear (quadratic) relation between those two quantities (solid blue curve). In practice, aberrations of the time-lens system are evaluated experimentally with a double-pulse experiment: the spacing in pixels between two pulses separated by a known delay is measured over the full acquisition window. This allows the determination of the time interval between adjacent pixels along the window (red line) and consequently, to retrieve the nonlinear mapping between temporal coordinate and horizontal axis of the camera (See Supplement for details on the calibration procedure and signal retrieval algorithm).

Before entering the description of the experiments and to facilitate the comprehension of our results, we describe in Fig. 2 a typical numerical simulation of the nonlinear Schrödinger equation (NLSE):

$$
i \frac{\partial \Psi}{\partial z}=\frac{\beta_{2}}{2} \frac{\partial^{2} \Psi}{\partial t^{2}}-\gamma|\Psi|^{2} \Psi
$$

where $\Psi$ is the envelope of the optical wavefield, $z$ is the longitudinal variable describing propagation distance, $t$ the time defined in the frame traveling at group velocity of the carrier wave, $\beta_{2}$ the group velocity dispersion coefficient at the carrier frequency, and $\gamma$ is the Kerr coefficient. Figure 2(a) shows as a $2 \mathrm{D}$ color plot the longitudinal evolution of a $\mathrm{cw}$ field initialy perturbed by realistic noise over $1 \mathrm{~km}$ on a temporal window of $150 \mathrm{ps}$ featuring MI. Parameters are chosen according to our experiments and are given in the caption of Fig. 2. Figure 2(a) illustrates how the initial noisy condensate evolves towards a quasi-regular pattern (between $400 \mathrm{~m}$ and $600 \mathrm{~m}$ ) before exhibiting a more complex spatio-temporal dynamics. The right panels highlight the temporal dynamics at two particular stages by showing both the intensity and the phase of the field at the initial state (Fig. 2(c)) and close to the so-called maximum compression point (MCP) of (Fig. 2(b)). The MCP can be defined in a statistical manner as the point where the Fourier spectrum is the broadest and appears as a saturation of the initial linear stage of MI (exponential growth of the spectral components) [6]. The initial state features small random fluctuations of intensity (blue curve) and uncorrelated fluctuations of the phase with very small excursion (red curve). On the other side, at the MCP, the field exhibits the formation of high intensity peaks separated by a duration close to the MI period $\left(T_{M I}=2 \pi \sqrt{\left|\beta_{2}\right| /\left(2 \gamma P_{0}\right)} \sim 7.1 \mathrm{ps}\right)$. Remarkably, the phase profile is now also highly structured and clear phase jumps of $\sim \pi$ are observed surrounding each of the high intensity peaks like, for instance, between $-50 \mathrm{ps}$ and $0 \mathrm{ps}$ ). This is reminiscent of the local appearance of structures that are close to solitons on finite background (SFB) also called breathers $[20,21]$. In the present case, the field observed in this window resembles the so-called Akhmediev breather $(\mathrm{AB})$ solution close to its $\mathrm{MCP}$. The $\mathrm{AB}$, whose intensity spatio-temporal dynamics is illustrated in Fig. 2(d), is a particular solution of Eq. (1) that is localised in $z$ but periodic in $t$ [22]. At its MCP (see Fig. 2(e)), it takes the form of regularly spaced peaks. In particular, consecutive zero intensity points are associated to a succession of $\pm \pi$ phase jumps giving rise to a periodic (square) phase profile (solid red curve) [23]. Recent experiments realised using fiber optics and photorefractive crystals have revealed traces of such ABs dynamics in the context of the seeded MI process [24-27]. We identify local reminiscent traces of this in our numerical simulation. Lastly, a common feature of the fundamental SFBs is that the sign of the phase flips when crossing the MCP (dashed red line is the phase just after the MCP). The stochastic nature of the noise-induced $\mathrm{MI}$ hinders the observation of the exact $\mathrm{AB}$ solution.

We have specifically designed an experiment to confirm the noteworthy features described in Fig. 2. Using the setup described in Fig. 1(a) the ultra-fast temporal dynamics of the field 


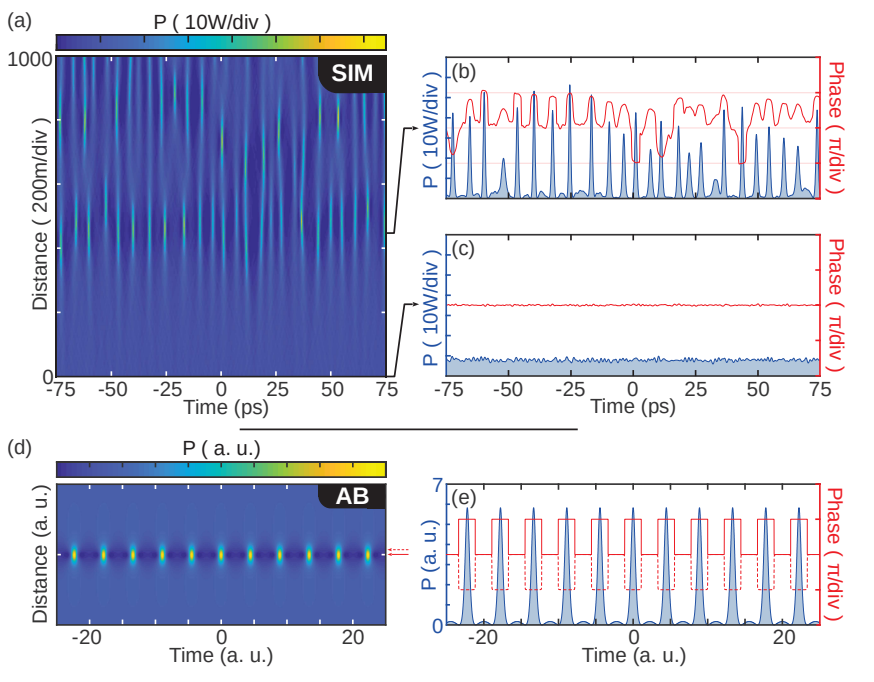

Fig. 2. Typical numerical simulation of the NLSE exhibiting noise-driven MI. (a) Spatio-temporal diagram of the intensity for the first $1000 \mathrm{~m}$ of propagation. Complex field at (c) the initial state and (b) after $420 \mathrm{~m}$ of propagation. $P_{0}=\left\langle|\Psi(z=0)|^{2}\right\rangle=6 \mathrm{~W}, \beta_{2}=-20 \mathrm{ps}^{2} / \mathrm{km}, \gamma=1.3 / \mathrm{W} / \mathrm{km}$. (d, e) Same layout as previously but for an exact AB solution. The dashed red line in (e) is the phase of the AB just after the maximum compression. Parameter for the AB is $a=0.25$ according to notation in Ref. [20].

can be accessed in single-shot at different stages of the MI process by varying the length of the fiber on the signal path (dashed box in Fig. 1(a)). In this letter we focus on the dynamics in the vicinity of the MCP which, for an input power of $6 \mathrm{~W}$, occurs after $\sim 500 \mathrm{~m}$ of propagation. This distance depends on the initial noise level within the MI gain bandwidth [28]. A convenient indicator to assess the randomness of the initial field is the normalized variance of the power $P=|\Psi|^{2}$ which is defined as $\sigma^{2}=\left(\left\langle P^{2}\right\rangle-\langle P\rangle^{2}\right) /\langle P\rangle^{2}$ where $\langle\cdot\rangle$ denotes average over time. We have evaluated a posteriori that in our experiments $\sigma_{0}=\sigma(z=0) \sim 0.07$. Note that we did not control the amount of noise in our system which mostly originates from the amplified spontaneous emission of the EDFA. The initial stage is conveniently accessed by removing the fiber spool and connecting the output of the EDFA directly to the detection apparatus. In each case, we have acquired 25000 frames which corresponds to a total of $5 \mu$ s effective recording duration.

Figure 3 shows selected, fully processed, experimental acquisitions at the initial stage (c) and after propagation in the $500 \mathrm{~m}$-long fiber (b). The temporal traces are interpolated in the spectral domain via the zero-padding technique to increase the sampling frequency by a factor 20. Intensity is normalised such that the average over all 25000 frames is 1. Overall, traces in Fig. 3 share strikingly resembling features with Figs. 2(b) and (c).

After propagation, we observed strongly modulated fields due to MI. The intensity profile exhibits, as expected, an overall irregular pattern where quasi-periodic structures can still be identified. This is in particular the case between $0 \mathrm{ps}$ and 50 ps where an almost regular train of pulses is observed. The period of this train is $\sim 7.4 \mathrm{ps}$ which in good agreement with the period associated to the frequency of maximum MI gain estimated from our experimental parameters ( $\sim 7.1 \mathrm{ps})$. Quite noteworthy are also the small "bumps" between each pulses

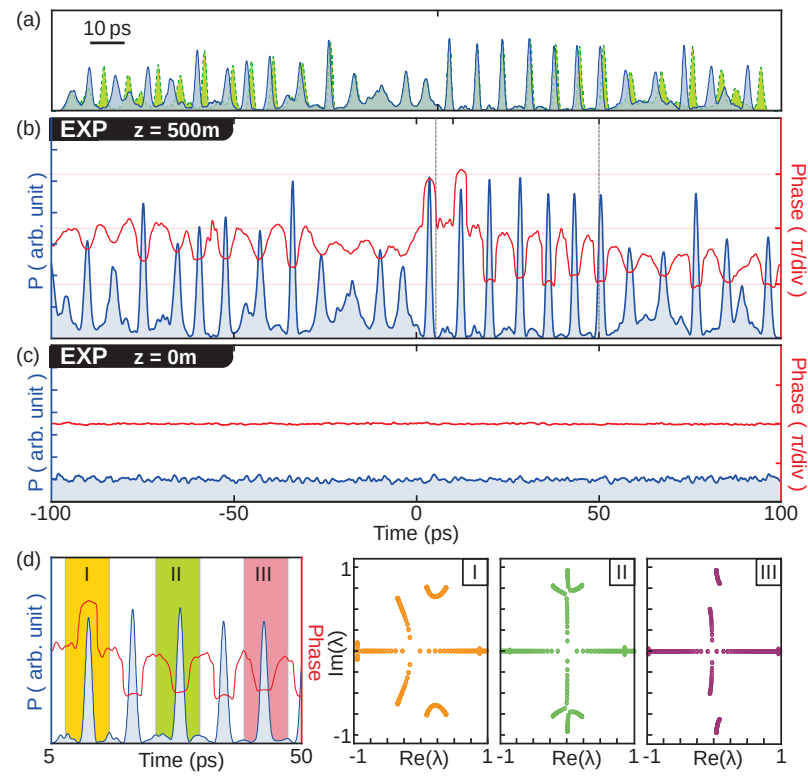

Fig. 3. Experimental complex fields recorded and reconstructed (c) before and (b) after propagation in $500 \mathrm{~m}$ of singlemode fiber. (a) shows the reconstructed intensity of the same field with (solid blue line) and without (dashed green line) compensation of the aberrations. (d) (left panel) Focus on the quasi-periodic pattern of (b) and (right panels) periodic IST profiles of isolated structures. Parameters are the same as in the caption of Fig. 2.

that are well captured by our recording system and which are also reminiscent of the $\mathrm{AB}$ solution (see Fig. 2(e)). Regarding the phase of the field, its structure is now strongly correlated to the intensity with large oscillations associated to high intensity peaks and rather flat profiles in regions where MI is not fully developed yet. In the region where the field is almost periodic, phase jumps close to $\pi$ are observed which is indicative of local proximity to the MCP. Sign flipping of the phase jumps between peaks are also observed in a similar fashion as the one observed in the numerical simulation. It is important to mention that, contrary to Fig. 2(b, c), the field in Fig. 3(c) is not related to the one in Fig. 3(b) as they come from different input pulses. Also, while the field presented in Fig. 3(b) has been selected among 25000 frames, the features that it exhibits are found on most of the frames, though the size of regular patterns typically vary between 2 and 10 structures.

Taking advantage of the simultaneous measurement of the phase and the amplitude reported in Fig. 3(b) we have performed the local nonlinear spectral analysis of some of the nonlinear structures observed in our experiment (see Fig. 3(d)). Following the methodology described in Ref. [29], we have isolated and analyzed the structures observed between $0 \mathrm{ps}$ and 50 ps and we show the result for 3 of them highlighted in the left panel. Remarkably each individual structure has a nonlinear spectrum (in the sense of the periodic inverse scattering transform (IST) method) composed of three bands (right panels), which means that it can be locally identified as representing a breather solution of the NLSE, see e.g. [27, 29].

Finally, Fig. 3(a) illustrates qualitatively the impact of the aberrations of the pump chirp on the reconstruction of the field. The superposition of the intensity of the field with and without 
(solid blue and dashed green lines resp.) correction of the aberrations shows that the cubic contribution to the phase of the pump is responsible for a stretching (squeezing resp.) of the time on the left (right resp.) side of the frame in accordance with the results shown in Fig. 1(c).

The direct measurement of the full complex field in the temporal domain over a $200 \mathrm{ps}-$ long window allows for the computation of the corresponding single-shot spectrum in the Fourier space with a comfortable $5 \mathrm{GHz}$ resolution. The span of the calculated spectrum is limited to $1 \mathrm{THz}$ by the 1 ps resolution of the SEAHORSE setup. Figure 4 shows the single-shot spectrum associated to the field of Fig. 3(b) (grey line) along with the average spectrum computed from the 25000 frames (blue line).

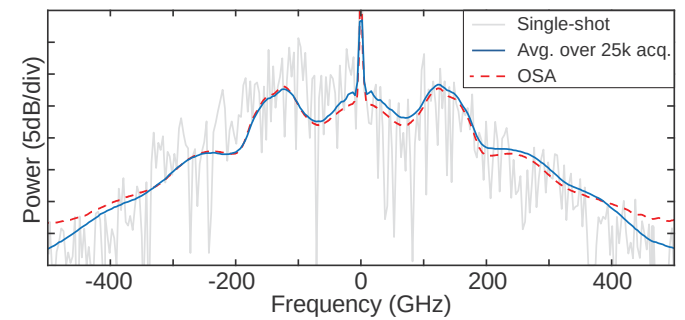

Fig. 4. Single-shot Fourier spectrum computed from the complex field of Fig. 3(b) along with the average spectrum computed over 25000 single-shot acquisitions and a trace from an optical spectrum analyzer (OSA) recorded simultaneously.

While the single-shot spectrum is inherently very noisy, the smoothing brought by the averaging allows clear observation of the standard MI sidebands at frequency detunings of $\sim \pm 125 \mathrm{GHz}$ (i.e. $\sim 8$ ps timescale). For comparison, the spectrum acquired using a standard optical spectrum analyzer (OSA) with $2 \mathrm{GHz}$ resolution directly at the output of the fiber is superimposed as a dashed red line and shows a very good agreement. The most noticeable discrepancy is found in the wings of the spectrum where the OSA trace presents higher power density than the spectrum computed from the SEAHORSE acquisitions. This can be ascribed to the fact that an optical signal is measured unnecessarily by the OSA during a large dead time lying between consecutive pulses. On the other hand, the time lens acquisitions are intrinsically performed over a temporal window fully comprised in the signal pulses, which results in a more accurate representation of the MI spectrum.

In summary, we report the single-shot observation of extended coherent structures, which are locally reminiscent of Akhmediev breathers, emerging in the nonlinear development of MI in fiber optics experiments. While intensity measurements covering 40 ps have been reported in the pioneering work of Ref. [11], the use of a heterodyne time-lens system enables here the simultaneous recording of both intensity and phase of the field over a very large temporal window of 200 ps allowing for observations with unprecedentedly great completeness. This enables reconstruction of the associated single-shot Fourier spectrum while also providing the opportunity to perform nonlinear spectral analysis of the observed structures.

Funding. European Regional Development Fund ( CPER Photonics for Society P4S); Ministry of Higher Education and Research, Hauts de France council; Agence Nationale de la Recherche (CEMPI ANR-11-LABX-0007, DYDICO ANR-16IDEX-0004).

\section{REFERENCES}

1. T. B. Benjamin and J. E. Feir, J. Fluid Mech. 27, 417 (1967).

2. T. Taniuti and H. Washimi, Phys. Rev. Lett. 21, 209 (1968).

3. K. E. Strecker, G. B. Partridge, A. G. Truscott, and R. G. Hulet, Nature 417, 150 (2002).

4. K. Tai, A. Hasegawa, and A. Tomita, Phys. Rev. Lett. 56, 135 (1986).

5. V. E. Zakharov and L. A. Ostrovsky, Phys. D: Nonlinear Phenom. 238, 540 (2009).

6. D. S. Agafontsev and V. E. Zakharov, Nonlinearity 28, 2791 (2015).

7. D. R. Solli, G. Herink, B. Jalali, and C. Ropers, Nat. Photonics 6, 463 (2012).

8. M. Närhi, L. Salmela, J. Toivonen, C. Billet, J. M. Dudley, and G. Genty, Nat. Commun. 9, 4923 (2018).

9. B. Wetzel, A. Stefani, L. Larger, P. A. Lacourt, J. M. Merolla, T. Sylvestre, A. Kudlinski, A. Mussot, G. Genty, F. Dias, and J. M. Dudley, Sci. Reports 2 (2012).

10. G. Herink, B. Jalali, C. Ropers, and D. R. Solli, Nat. Photonics 10, 321 (2016).

11. M. Närhi, B. Wetzel, C. Billet, S. Toenger, T. Sylvestre, J.-M. Merolla, R. Morandotti, F. Dias, G. Genty, and J. M. Dudley, Nat. Commun. 7, ncomms13675 (2016).

12. P. Suret, R. E. Koussaifi, A. Tikan, C. Evain, S. Randoux, C. Szwaj, and S. Bielawski, Nat. Commun. 7, 13136 (2016).

13. A. E. Kraych, P. Suret, G. El, and S. Randoux, Phys. Rev. Lett. 122, 054101 (2019).

14. A. E. Kraych, D. Agafontsev, S. Randoux, and P. Suret, Phys. Rev. Lett. 123, 093902 (2019).

15. C. Dorrer, Opt. Lett. 31, 540 (2006).

16. P. Ryczkowski, M. Närhi, C. Billet, J.-M. Merolla, G. Genty, and J. M. Dudley, Nat. Photonics 12, 221 (2018).

17. A. Tikan, S. Bielawski, C. Szwaj, S. Randoux, and P. Suret, Nat. Photonics 12, 228 (2018).

18. C. V. Bennett and B. H. Kolner, Opt. Lett. 24, 783 (1999).

19. C. Bennett and B. Kolner, IEEE J. Quantum Electron. 37, 20 (2001).

20. J. M. Dudley, F. Dias, M. Erkintalo, and G. Genty, Nat. Photonics 8, 755 (2014).

21. S. Toenger, T. Godin, C. Billet, F. Dias, M. Erkintalo, G. Genty, and J. M. Dudley, Sci. Reports 5, 10380 (2015).

22. N. N. Akhmediev and V. I. Korneev, Theor. Math. Phys. 69, 1089 (1986).

23. A. Tikan, C. Billet, G. El, A. Tovbis, M. Bertola, T. Sylvestre, F. Gustave, S. Randoux, G. Genty, P. Suret, and J. M. Dudley, Phys. Rev. Lett. 119 (2017).

24. A. Mussot, C. Naveau, M. Conforti, A. Kudlinski, F. Copie, P. Szriftgiser, and S. Trillo, Nat. Photonics 12, 303 (2018).

25. C. Naveau, P. Szriftgiser, A. Kudlinski, M. Conforti, S. Trillo, and A. Mussot, Opt. Lett. 44, 763 (2019).

26. D. Pierangeli, M. Flammini, L. Zhang, G. Marcucci, A. Agranat, P. Grinevich, P. Santini, C. Conti, and E. DelRe, Phys. Rev. X 8, 041017 (2018).

27. G. Marcucci, D. Pierangeli, A. J. Agranat, R.-K. Lee, E. DelRe, and C. Conti, Nat. Commun. 10, 5090 (2019).

28. J. Soto-Crespo, N. Devine, and N. Akhmediev, Phys. Rev. Lett. 116, 103901 (2016).

29. S. Randoux, P. Suret, and G. El, Sci. Reports 6, 29238 (2016). 


\section{Single-shot observation of breathers from noise-induced modulation instability using heterodyne temporal imaging: supplemental document}

The purpose of this Supplemental Material is to provide some details about the methods used to extract the amplitude and the phase of the optical signal from the raw data recorded in the experiments. In the first demonstration of the SEAHORSE (spatial encoding arrangement with hologram observation for recording in single shot the electric field), the field of view was a few tens of picoseconds and aberrations were neglected [1].

In this Letter, we extend the field of view up to 200 ps and we demonstrate that aberrations induced by the third order dispersion can be significantly compensated in the data analysis. The theoretical background needed to describe the operation of the SEAHORSE is briefly given in Sec. I. Details about numerical data processing and aberration compensation are provided in Sec. II.

\section{SIGNAL RETRIEVAL IN THE SEAHORSE (THEORY)}

The notations used throughout this Supplementary document for the field and the Fourier transform are listed below.

$$
\begin{aligned}
& \text { Direct Fourier Transform } F T(A(t))=\tilde{A}(\omega)=\int_{-\infty}^{+\infty} A(t) e^{-i \omega t} d t \\
& \text { Inverse Fourier Transform } F T^{-1}(\tilde{A}(\omega))=A(t)=\frac{1}{2 \pi} \int_{-\infty}^{+\infty} \tilde{A}(\omega) e^{i \omega t} d \omega \\
& \text { Convolution: } \quad A \otimes B(\omega)=\int_{-\infty}^{+\infty} A\left(\omega^{\prime}\right) B\left(\omega-\omega^{\prime}\right) d \omega^{\prime}
\end{aligned}
$$

$S(t)$ is the slowly-varying amplitude of the electric field $e(t)=S(t) e^{i\left(\omega_{0} t\right)}+$ c.c. under study:

$$
\begin{aligned}
& s(t)=|S(t)| \\
& s(t)=s(t) e^{i \phi(t)}
\end{aligned}
$$

The slowly-varying amplitude of the pump pulse is $P(t)$. The Fourier components of the chirped pump pulse read :

$$
\begin{aligned}
p(\omega) & =|\tilde{P}(\omega)| \\
\tilde{P}(\omega) & =p(\omega) e^{-i \tilde{\phi}_{P}(\omega)} \\
\tilde{\phi}_{P}(\omega) & =\frac{B_{2}}{2 !} \omega^{2}+\frac{B_{3}}{3 !} \omega^{3}
\end{aligned}
$$

$B_{2}$ is the group velocity dispersion (GVD) experienced by the pump pulse in the regenerative amplifier (Astrella, Coherent)

$B_{3}$ is the third order dispersion (TOD) experienced by the pump pulse.

In the BBO crystal, the pump intensity is much higher than the signal intensity. In the approximation of weak conversion, the field generated by sum frequency generation (SFG) is proportional to $P(t)$ and $S(t)[2]$

$$
S F G(t) \propto S(t) P(t)
$$

In the SEAHORSE, a monochromatic reference beam is also launched into the BBO crystal and the SFG between the pump and the reference provides a field that is simply proportional to $P(t)$ : 
$S F G_{R}(t) \propto P(t)$.

The second part of the SEAHORSE is made of a single-shot spectrum analyser. We thus measure the modulus of $\widetilde{S F G}(\omega)$ and the relative phase between $\widetilde{S F G}(\omega)$ and $\widetilde{S F G_{R}}(\omega)$.

Finally, the recorded data $\tilde{X}(\omega)$ thus reads :

$$
\tilde{X}(\omega)=[\tilde{S}(\omega) \otimes \tilde{P}(\omega)] e^{i \tilde{\phi}_{P}(\omega)}
$$

$$
\begin{aligned}
& \tilde{X}(\omega)=\int_{-\infty}^{+\infty} \tilde{S}\left(\omega^{\prime}\right) p\left(\omega-\omega^{\prime}\right) \exp \left[-i \frac{B_{2}}{2}\left(\omega-\omega^{\prime}\right)^{2}-i \frac{B_{3}}{6}\left(\omega-\omega^{\prime}\right)^{3}\right] \exp \left[+i \frac{B_{2}}{2} \omega^{2}+i \frac{B_{3}}{6} \omega^{3}\right] d \omega^{\prime} \\
& \tilde{X}(\omega)=\int_{-\infty}^{+\infty} \tilde{S}\left(\omega^{\prime}\right) p\left(\omega-\omega^{\prime}\right) \exp \left[+i B_{2} \omega \omega^{\prime}-i \frac{B_{2}}{2} \omega^{\prime 2}+i \frac{B_{3}}{2} \omega^{2} \omega^{\prime}-i \frac{B_{3}}{2} \omega \omega^{\prime 2}+i \frac{B_{3}}{6} \omega^{\prime 3}\right] d \omega^{\prime}
\end{aligned}
$$

Parameters of the experiments

$$
\begin{aligned}
B_{2} & =1.437 \mathrm{ps}^{2} \\
B_{3} & =-2.68810^{-3} \mathrm{ps}^{3} \\
\omega_{\text {max }} \text { of the pump } & \approx 2 \pi \times 7 \mathrm{THz} \\
\omega_{\text {max }}^{\prime} \text { of the signal } & \approx 2 \pi \times 0.2 \mathrm{THz} \\
B_{3}\left(\omega_{\text {max }}^{\prime}\right)^{3} / 6 & \approx 9.10^{-4} \\
B_{3}\left(\omega_{\text {max }}^{\prime}\right)^{2} \omega_{\text {max }} / 2 & \approx 0.09
\end{aligned}
$$

We neglect the last two terms in the integral. Moreover, as $\omega_{\max } \gg \omega_{\max }^{\prime}$ one can approximate $\tilde{p}\left(\omega-\omega^{\prime}\right) \approx \tilde{p}(\omega)$. Finally, one gets :

$$
\tilde{X}(\omega)=p(\omega) \int_{-\infty}^{+\infty} \tilde{S}\left(\omega^{\prime}\right) \exp \left[-i \frac{B_{2}}{2} \omega^{\prime 2}\right] \exp \left[+i\left(B_{2} \omega+\frac{B_{3}}{2} \omega^{2}\right) \omega^{\prime}\right] d \omega^{\prime}
$$

Assuming stationarity of the statistics of the random signal $S(t)$, the Fourier components are $\delta$-correlated : $\left\langle\tilde{S}\left(\omega^{\prime}\right) \tilde{S}^{*}\left(\omega^{\prime \prime}\right)\right\rangle=N\left(\omega^{\prime}\right) \delta\left(\omega^{\prime}-\omega^{\prime \prime}\right)$. By averaging the data over many realizations, we measure $\tilde{p}(\omega)$ :

$$
p(\omega)=\sqrt{\frac{\left\langle|\tilde{X}(\omega)|^{2}\right\rangle}{P_{0}}} \text { where } P_{0}=\int N(\omega) d \omega
$$

We define

$$
\begin{aligned}
\tilde{X}^{\prime}(\omega) & =\tilde{X}(\omega) / \tilde{p}(\omega) \\
t & =B_{2} \omega+\frac{B_{3}}{2} \omega^{2} \\
Y(t) & =\tilde{X}[\omega(t)]
\end{aligned}
$$

Eq. (S6) finally reads:

$$
\left.Y(t)=\int_{-\infty}^{+\infty} \tilde{S}\left(\omega^{\prime}\right) \exp \left[-i \frac{B_{2}}{2} \omega^{\prime 2}\right] \exp \left[+i \omega^{\prime} t\right] d \omega^{\prime}\right)
$$

\section{Final data analysis}

Starting from Eq.(S11), the signal $S(t)$ is retrieved by using the simple algorithm:

1/ Compute $\tilde{Y}(\omega)$, the Fourier transform of $Y(t)$

$2 /$ Apply the operator of propagation $e^{+i B_{2} \omega^{2} / 2}$

3 / Perform the inverse Fourier transform 
It is important to emphasize that in this algorithm, the influence of $B_{3}$ on the spectral encoding of time $t(\omega)=B_{2} \omega+\frac{B_{3}}{2} \omega^{2}$ is compensated. On the contrary, we neglect here the influence of $B_{3}$ on the phases in the integral. The study of these aberrations in the case of signal with broader spectrum is beyond the scope of this Letter and this point deserves further investigations.

\section{NUMERICAL PROCESSING AND ABERRATIONS COMPENSATION :}

\section{A. Calibration of time}

The SEAHORSE technique is based on the spectral encoding of the signal. The spectral profile of the SFG signal is observed on a sCMOS camera where the frequency components are equally spaced on the $\mathrm{x}$ axis by means of a simple grating. A given pixel $p_{x}$ of the horizontal axis corresponds to a given frequency $\omega$ and thus to a given time through Eq. (S9).

The first step of the analysis is to find the quantitative relationship between time and the index $p x$ of the pixel:

$$
t=a\left(p x-p x_{0}\right)+\frac{b}{2}\left(p x-p x_{0}\right)^{2}
$$

where $a \propto B_{2}$ and $b \propto B_{3}$.

$a$ and $b$ are measured by using a double pulse experiment. Two pulses separated by $81.6 \mathrm{ps}$ are generated by launching a picosecond mode-locked laser (Pritel) beam into a Michelson interferometer. The two pulses are recorded by using the SEAHORSE device. Even though the time interval between the two pulses is fixed at the output of the Michelson interferometer, the pixel interval between the two recorded pulses depends on their positions in the window of measurement because $b \neq 0$.

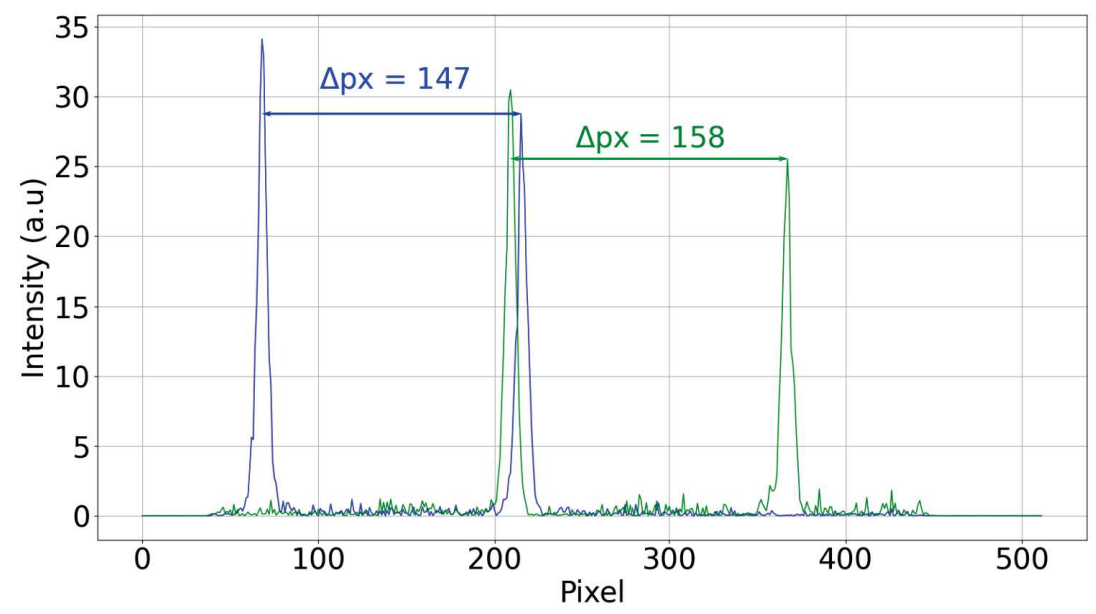

Fig. S1. Double pulse recorded by using the SEAHORSE. The separation $\Delta p x$ between the two pulses recorded on the camera depends on their mean position $\langle p x\rangle$ within the measurement window. Blue and green curve are two different snapshots.

As an example, two different snapshots of the double pulses recorded by using the SEAHORSE are plotted in Fig. S1. By using numerous recorded frames, we measure the distance in pixel between the two pulses as a function of their mean position $\langle p x\rangle$ on the camera (see the blue curve in Fig. S2).

By using an optimization procedure, we evaluate $p x_{0}, a$ and $b$ of Eq. (S12) for which the measured interval $\Delta t$ remains constant over the whole window of observation (see the red curve in Fig. S2): 


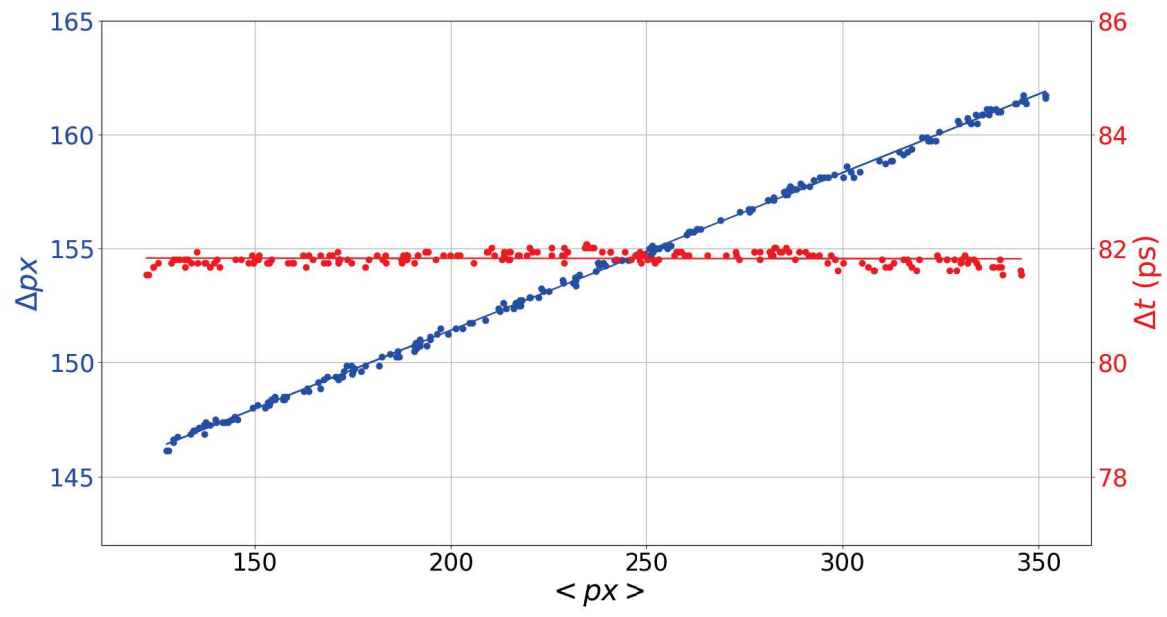

Fig. S2. Blue dot : number of pixels $\Delta p x$ between the centroids of the two pulses as a function of their mean position $\langle p x\rangle$ in the window. Red dot : time interval between the two pulses $\Delta t$ computed from the nonlinear pixel to time conversion Eq. (S12). Blue and red solid lines are linear fits related to the same color.

$$
\begin{aligned}
a & =5.27 * 10^{-1} \mathrm{ps} / \text { pixel } \\
b & =-1.19 * 10^{-4} \mathrm{ps} / \mathrm{pixel}^{2} \\
p x_{0} & =232
\end{aligned}
$$

Note that, as expected, the evaluated value of $p x_{0}$ is close to the centroid of the signal. Note finally that the optimization procedure is an iterative process taking into account the digital focus (see below).

\section{B. Signal retrieval}

Above, the correspondence between the time $t$ and the index $p x$ of the horizontal pixel of the camera has been established.

In a second step, the intensity and phase of $Y(t)$ [see Eq. (S11)] are retrieved from the raw data recorded onto the camera. A typical single-shot pattern recorded by the camera is plotted in Fig. S3.a). Roughly speaking, for a given time, the intensity $|Y(t)|^{2}$ corresponds to the sum of the pattern over a vertical line divided by $p(\omega)$ [see Fig. S3.b)]. The phase is encoded in the fringes positions and is retrieved by means of Fourier transform [see Fig. S3.d)]. See [1] for a detailed description of the full procedure.

In a last step, we perform the Final data analysis described in Sec. 1. Conceptually, this step corresponds to a digital focus analogous in the time domain to the spatial digital holography. The intensity and phase of the retrieved signal is plotted in Fig.S3.(e,f).

It is important to note that the influence of the third order dispersion in the spectral encoding of time is rigorously compensated in the data analysis. On the contrary, for the signals under investigation in this Letter, the influence of the third order dispersion in the digital focus is neglected. The compensation of aberrations for signals with broader spectrum deserves further investigations. 
a)
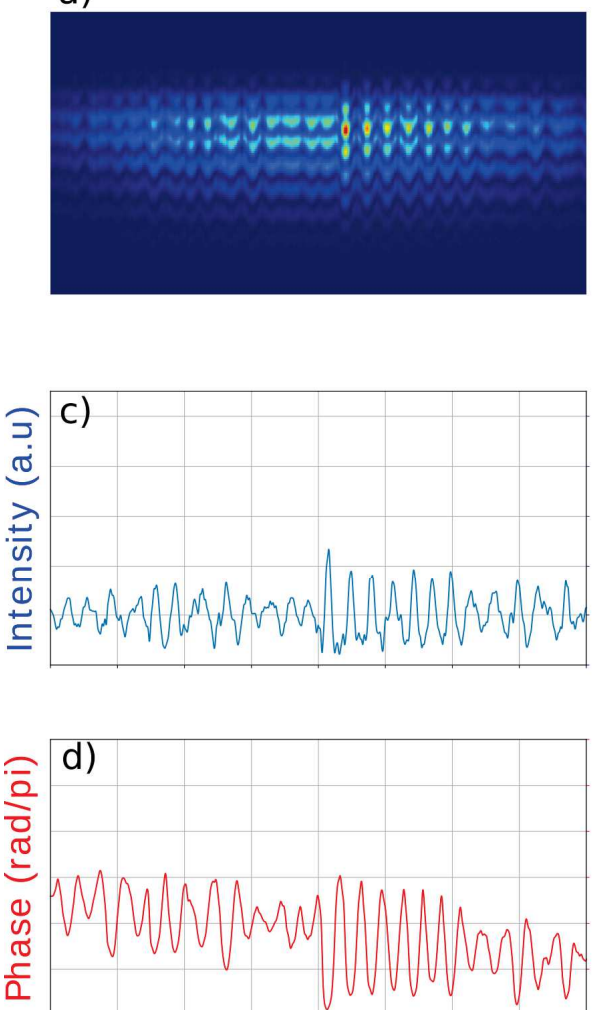

$-100-50$ b)

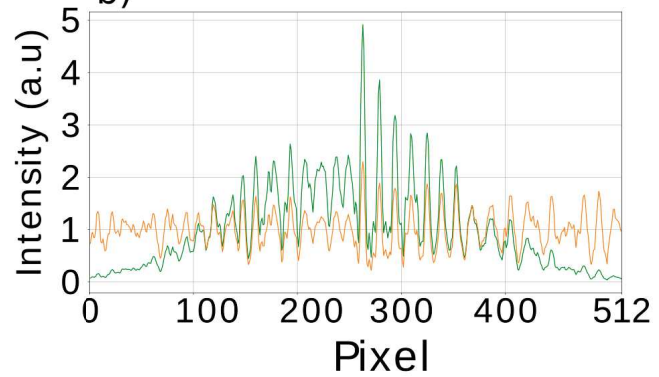

3.0

2.0

1.0

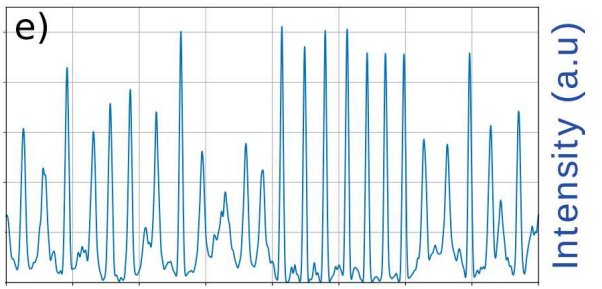

\section{胥}

0.0

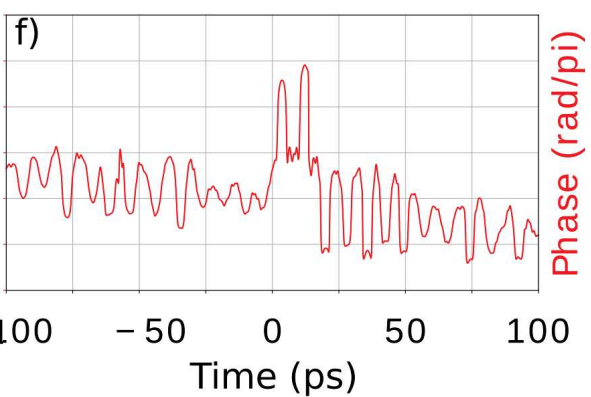

$S(t)$

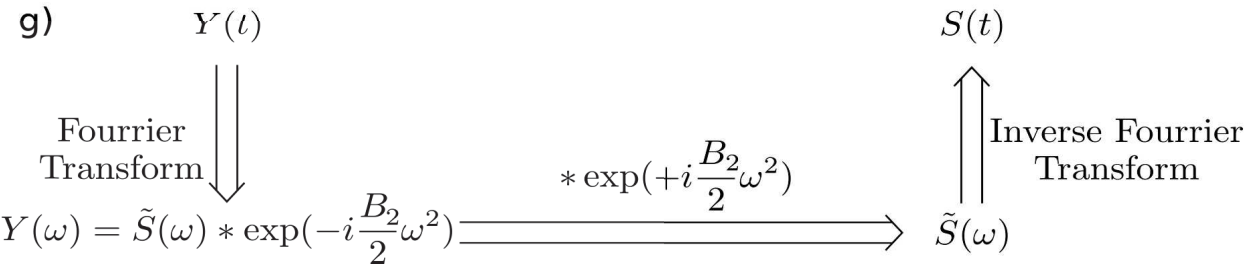

Fig. S3. a) Typical single-shot pattern recorded by the camera. b) Green line : intensity profile extracted from a). Orange : intensity profile divided by the averaged envelop $p(\omega)$. c) Intensity $|Y(t)|^{2}$ d) Phase of $Y(t)$. In c) and d) the nonlinear pixel to time conversion of Eq. (S12) has been used. e) Intensity of the studied signal $|S(t)|^{2}$ f) Phase of $S(t)$ g) Principle of the digital focus enabling to retrieve $(e, f)$ from $(c, d)$.

\section{REFERENCES}

1. A. Tikan, S. Bielawski, C. Szwaj, S. Randoux, and P. Suret, "Single-shot measurement of phase and amplitude by using a heterodyne time-lens system and ultrafast digital time-holography," Nat. Photonics 12, 228-234 (2018).

2. R. W. Boyd, Nonlinear optics (Academic Press, Amsterdam ; Boston, 2008), 3rd ed. 